\title{
Saneamiento Ambiental y Participación Ciudadana
}

\author{
Autor: José Antonio Peña Barreto \\ Universidad Pedagógica Experimental Libertador, UPEL \\ joseantbarreto2014@gmail.com \\ Barinas, Venezuela
}

\section{Resumen}

El presente trabajo de investigación se plantea con el propósito de proponer un plan de acción para el saneamiento ambiental con la participación ciudadana en la Escuela Básica Nacional Concentrada "Manuela Sáenz" de la parroquia Ciudad Bolivia del Municipio Pedraza Estado Barinas. Se inscribe en el enfoque cualitativo, bajo la modalidad de investigación acción participativa y sustentada en un diseño descriptivo narrativo. El escenario seleccionado para realización del trabajo fue la Escuela Nacional Concentrada "Manuela Sáenz", los informantes claves están constituidos por 1 miembro de la comunidad, 1 miembro del consejo comunal y 1 docente de la institución. Las técnicas utilizadas son la observación y la entrevista a profundidad, usando como instrumentos un cuaderno de campo y un guion de entrevista. Para la presentación y análisis de resultados se categorizó, trianguló y teorizó la información obtenida, realizando un diagnostico profundo y detallado sobre el manejo integral de los residuos sólidos. Posteriormente se elaboró la propuesta denominada Plan de acción para el saneamiento ambiental con la participación ciudadana en la Escuela Básica Nacional Concentrada Manuela Sáenz. Luego se ejecutó la propuesta en donde los participantes, opinaron que las actividades realizadas permitieron obtener conocimientos básicos muy importantes sobre el saneamiento ambiental.

Palabras clave: saneamiento ambiental; plan acción; contaminación ambiental.

Fecha de Recepción: 12-07-2016 Fecha de Aceptación: 28-07-2016 Sanitation and Civic Participation 


\begin{abstract}
This research is proposed in order to propose an action plan for environmental sanitation citizen participation in the "Manuela Sáenz" Concentrating National Basic School Parish Municipality City Bolivia Pedraza Barinas state. It is part of the qualitative approach in the form of participatory action research and supported by a narrative descriptive design. The selected scenario for performance of work was the Concentrating National School "Manuela Sáenz" key informants consist of 1 member of the community, 1 member of the community council and one teacher of the institution. The techniques used are observation and depth interview, using as instruments a field notebook and an interview script. For the presentation and analysis of results he was categorized, triangulated and theorized the information obtained, performing a thorough and detailed report on the integrated management of solid waste diagnosis. Subsequently, the proposal called Action Plan for environmental sanitation was developed with citizen participation in concentrated Manuela Saenz National Basic School. Then the proposal where participants expressed the view that activities allowed to obtain very important basic knowledge on environmental sanitation was run.
\end{abstract}

\begin{abstract}
Keywords: environmental sanitation; action plan; environmental pollution.
\end{abstract}

Date Received: 12-07-2016 1. A modo de Introducción

Date Acceptance: 28-07-2016 
En los últimos tiempos el planeta tierra ha experimentado cambios, producidos por las diversas actividades humanas. El hombre ha creado desequilibrios ambientales en su ambición de obtener mayores riquezas. En la mayoría de las ciudades se observa el problema de la disposición de los residuos sólidos; la contaminación ha aumentado, producto del agotamiento desmedido de los recursos naturales. La sociedad actual está muy ligada a un estilo de vida confortable característico de la modernidad, donde impera el consumismo. Cada día crece más la acumulación de residuos domésticos e industriales, necesitándose mayor cantidad de recursos físico y humanos para combatir el problema de la basura. La cual es definida por Pérez (2011) de la siguiente manera:

La basura es la acumulación de residuos sólidos producidos por el hombre en sus actividades diarias. Esos residuos están constituidos por desechos sólidos de origen orgánico, animal o vegetal; o inorgánicos como son los pedazos de vidrios, de plástico, de metal, escombros, etc. Según de donde provengan su nombre recibirá el nombre de su origen: domestico, industrial o comercial, etc. (pág. 12).

De acuerdo, con esta definición la basura es producto de la actividad humana, por lo tanto es tarea del hombre realizar acciones para disminuir la producción de residuos sólidos, efectuando de esta manera labores para mejorar el medio ambiente. La contaminación ambiental por desechos domésticos e industriales son focos donde proliferan plagas que son vectores de enfermedades. En este sentido una de las actividades más efectivas para ofrecer una mejor calidad de vida es el saneamiento ambiental. Según Pérez (ob.cit.), "consiste en el mantenimiento de los elementos del medio ambiente (tanto naturales como aportados por el hombre) en condiciones aptas para el desarrollo del ser humano tanto en lo individual como en lo colectivo" (pág. 13). En este orden de ideas, es necesario que la sociedad tome conciencia acerca de la importancia de mantener un ambiente apto para la vida sana. La 
salud es primordial para el desarrollo de las personas, y contribuye a su autorrealización, requerido para la formación de una sociedad prospera y con valores ambientales.

A nivel mundial el saneamiento ambiental ha constituido una buena alternativa para mejorar la calidad del ambiente, según Lake (2007), "Para poder avanzar en materia ambiental es necesario lograr una amplia cooperación mediante alianzas públicas y privadas, la participación de las comunidades y el aumento del grado de conciencia de la población en general" (pág. 1). Con respecto a estas afirmaciones realizadas por el secretario ejecutivo del Fondo para la Infancia de las Naciones Unidas (UNICEF) la participación de la comunidad organizada es importante para unir esfuerzos con el fin de lograr bienes comunes. Por esta razón, las jornadas de sensibilización ambiental son imprescindibles para forma ciudadanos con valores de solidaridad con el ambiente y por ende con la salud de la colectividad.

Estudios realizados han demostrado que existen comunidades que no poseen las condiciones de salud necesarios para tener una buena calidad de vida. Según el informe de la Conferencia Latinoamericana de Saneamiento (2007) "más de 2.500 millones de personas $41 \%$ de la población mundial no tiene acceso a la calidad de vida saludable que solo el saneamiento puede ofrecerles" (pág. 2). De acuerdo a estas estadísticas es esencial la coordinación de los entes públicos con el sector privado para lograr las alianzas que contribuyan con la construcción de infraestructuras óptimas para mantener los ambientes sanos.

Por otro lado, si se observan los indicadores de la pobreza se encuentra la falta de saneamiento ambiental, la cual se traduce en poblaciones con altos índices de enfermedades epidémicas y endémicas que merman el desarrollo tanto individual como colectivo de las comunidades. Con relación a esta temática, la Conferencia Latinoamericana de Saneamiento (ob. cit) afirma: 
Son aún más de 120 millones los latinoamericanos que carecen de acceso a sistemas mejorados de saneamiento. Menos del $15 \%$ de las aguas residuales son tratadas en el continente. $Y$ la evidencia ha demostrado que donde no está disponible ninguna instalación sanitaria ni sistemas de disposición de residuos sólidos, las aguas residuales permanecen en el lugar que las personas habitan, y los grupos más pobres y vulnerables son los que más sufren (pág. 5).

Con respecto a este dato, se evidencia que el problema del saneamiento ambiental debe ser considerado como una prioridad por los entes gubernamentales y las organizaciones comunitarias y no convertir esta necesidad de los pueblos en ofertas electorales, que al final se quedan solo en promesas.

En observaciones realizadas de forma empírica en la Escuela Básica Nacional Concentrada "Manuela Sáenz" se detectó la necesidad de mejorar las condiciones ambientales, esta institución presenta aguas estancadas producto de las lluvias, así como también maleza lo cual constituye el criadero de plagas tales como cucarachas, ratas, zancudos, avispas, culebras, entre otros, convirtiéndose en focos de enfermedades para los alumnos de esta unidad educativa, además de intervenir en el desarrollo de las actividades de educación física deporte y recreación. De allí la importancia de la participación ciudadana para la elaboración y ejecución de proyectos que mejoren las condiciones de saneamiento ambiental del plantel.

El presente proyecto de investigación está orientado a abordar la política de saneamiento ambiental del terreno y áreas verdes de la Escuela Básica Nacional Concentrada "Manuela Sáenz" ubicada en la urbanización la Floresta, parroquia Ciudad Bolivia del Municipio Pedraza del Estado Barinas. Con el presente estudio se pretende organizar a la comunidad para establecer un comité de mantenimiento que garantice la calidad del ambiente para los 
estudiantes y todas las personas que forman parte del quehacer educativo de manera sustentable.

A su vez, plantea la promoción de una cultura sanitaria dentro de las comunidades la Floresta, Girasoles, 24 de Julio y 13 de Enero, y el fortalecimiento de las organizaciones comunitarias en la gestión local del saneamiento ambiental. Además la estrategia de sustentabilidad se fundamenta en la integración de las organizaciones comunitarias como los Consejos Comunales, autoridades locales y sectoriales.

\subsection{Propósito de la investigación}

Promover un plan de acción para el saneamiento ambiental con la participación ciudadana en la Escuela Básica Nacional Concentrada "Manuela Sáenz" de la parroquia Ciudad Bolivia del Municipio Pedraza Estado Barinas.

\subsection{Antecedentes}

Dentro de los estudios considerados como relevantes para este estudio se encuentra el de López (2012), trabajo investigativo realizado en la Universidad Politécnica Territorial del Oeste del Estado Sucre "Clodosbaldo Russián" titulado Plan de acción para la formación ambiental comunitaria. Este trabajo comunitario tiene el propósito Sensibilizar sobre el saneamiento ambiental y conservación del balneario de la comunidad de Rio Guarapiche. Esto bajo un diseño de investigación acción participativa, se construyó una estructura metodológica, la cual contempló las siguientes fases del proyecto diagnóstico participativo, planificación de acción y bitácora de las actividades realizadas. Esto conllevo a llegar a la siguiente conclusión; por medio de la participación protagónica y participativa de la comunidad se consigue establecer relaciones de alto valor de responsabilidad y corresponsabilidad en la administración comunitaria, hecho un desarrollo sostenible, amigable con el ambiente. Este estudio presenta una metodología acorde con la 
investigación en curso aportando una experiencia enriquecedora y una extraordinaria fuente documental.

Otra de las investigaciones consideradas como antecedente es la de Torrealba (2012) quien realizo un trabajo en la Universidad Bolivariana de Venezuela, el cual se denominó Plan de saneamiento de la Quebrada "Las Piedras" del Barrio Colombia Sur ubicado en el Municipio Guanare del Estado Portuguesa. La investigación estuvo sustentada en un proyecto acción, el cual la Al mismo tiempo, la investigación se ubicó como de campo debido que los datos fueron obtenidos directamente de la realidad Tiene por finalidad la promoción y el mejoramiento de condiciones de vida urbana y rural. Significa trabajar en la conservación de la salud de la población y juega un papel importante en la prevención de las enfermedades diarreicas cuyo origen está vinculado con deficiencias en la limpieza de las comunidades. Esta investigación planteo la realidad de una colectividad que al igual que otra enfrenta el problema de la contaminación ambiental, lo que implica que el saneamiento surge como una alternativa fundamental para garantizar un ambiente de calidad, para el estudio que se está realizando la metodología y producción teórica representan una guía fundamental para su desarrollo.

\section{Teoría y Conceptos}

Desde la visión de la filosofía, la presente investigación está orientada en el marco del enfoque constructivista, el cual permite desarrollar estrategias que tienen su origen las vivencias de las personas relacionadas en el proceso, según Moraga (2011):

Básicamente puede decirse que el constructivismo es el modelo que mantiene que una persona, tanto en los aspectos cognitivos, sociales y afectivos del comportamiento, no es un mero producto del ambiente ni un simple resultado de sus disposiciones internas, sino una construcción propia que se va produciendo día a día como resultado de la interacción de estos 
dos factores. En consecuencia, según la posición constructivista, el conocimiento no es una copia de la realidad, sino una construcción del ser humano, esta construcción se realiza con los esquemas que la persona ya posee (conocimientos previos), o sea con lo que ya construyó en su relación con el medio que lo rodea (pág. 1).

De acuerdo con esta definición, la teoría constructivista es ideal para promover la vinculación comunitaria en el saneamiento ambiental, lo que representa la integración de los conocimientos previos de los habitantes de la comunidad en estudio con las nuevas situaciones que permitirán la construcción de aprendizajes significativos.

\subsection{Saneamiento ambiental}

Desarrollar el concepto de saneamiento ambiental es necesario para entender la dinámica entre la interacción del ser humano y el ambiente que comparte con el resto de los seres vivos. Brindar un ambiente de calidad es importante para vida saludable, razón por la cual todas las personas tienen derecho a un ambiente agradable y limpio. Con respecto a la temática Suarez (2012):

Saneamiento es el proceso mediante el cual se identifican y evalúan factores de riesgo sobre la salud, condicionados por actitudes y prácticas inadecuadas a nivel familiar, comunitario y regional. Dicho diagnostico pretende establecer y priorizar esta problemática para su atención. El saneamiento ambiental es una función de la Salud Pública cuyo propósito es controlar, disminuir o eliminar riesgos derivados desiertas condiciones del ambiente físico y social que tienen el potencial de afectar a la salud; proporcionando a su vez confort al individuo y al colectivo (pág. 1).

De acuerdo con lo expuesto anteriormente, el saneamiento ambiental busca disminuir los factores de riesgo que afecten la calidad de vida de los ciudadanos. Sin embargo, es de gran importancia realizar un buen diagnóstico 
para coordinar las acciones esenciales para lograr un buen proceso de higiene integral. Con relación al tema la Conferencia Latinoamericana de Saneamiento (2007) plantea que "El mejoramiento de los servicios de saneamiento y las buenas prácticas de higiene contribuyen significativamente al bienestar del ser humano a través de la protección de la salud, la conservación del medio ambiente y reducción de la pobreza" (pág. 2). En este sentido, es necesario garantizar a la población condiciones higiénicas que permitan desarrollar una vida plena en un ambiente sin contaminación.

\subsection{Estrategias para el saneamiento ambiental}

El saneamiento ambiental, requiere de estrategias para que sea oportuno, eficiente y esté conectado con la cultura ciudadana, es importante que las personas estén integradas a sus propios procesos sociales. Con relación al tema Suarez (ob.cit.) plantea:

Educación y concientización ambiental, a fin de incorporar a la población a la participación responsable en la toma de decisiones, asegurar la retransmisión de conceptos en el nivel de complejidad adecuado al destinatario. Estimulación del desarrollo de modelos de comunicación de riesgos, que aseguren la comprensión de la población sobre la interacción entre diferentes riesgos para la salud (pág. 3).

En este sentido, es trascendental que el proceso educativo esté presente en la formación de los ciudadanos, el saneamiento es bueno para mantener los espacios limpios y saludables, sin embargo, sin la participación comunitaria no tiene sentido alguno, se requiere de un buen sentimiento de pertenencia y preocupación por el ambiente que es de todos y al forman parte todos los seres vivos.

\subsection{Saneamiento ambiental y el desarrollo de la higiene}


El saneamiento es motivo de preocupación a nivel mundial, y día tras día se demostrado que es una necesidad fundamental garantizar una vida saludable, Según la Organización Mundial de la Salud (2014): "Aproximadamente 2,4 mil millones de personas en todo el mundo viven en condiciones insalubres. Sus prácticas de higiene son tan malas que su nivel de exposición a riesgos de incidencia y diseminación de enfermedades infecciosas es altísimo". (pág. 1). De acuerdo a esta estadística el saneamiento contribuye con el control de enfermedades disminuyendo los factores de riesgo.

\subsection{Participación ciudadana}

En la actualidad la participación de los ciudadanos para resolver problemas comunes es una actividad necesaria que busca la sensibilización de las personas. El hombre es un ser social por naturaleza, no puede vivir aislado, sin embargo esta integración siempre ha estado condicionada por intereses individuales; situación que ha venido cambiando, debido a que los problemas tanto sociales, como ambientales afectan a todas las sociedades y se requiere de la participación de todos los seres humanos. Según Ceballos (2007):

La participación ciudadana es comprendida como un proceso social, continuo y dinámico, por medio del cual los miembros de una comunidad a través de mecanismos establecidos y organizaciones legítimas en las cuales se encuentren representadas todos los miembros de la comunidad, pues resulta difícil dialogar con todos y cada uno de los miembros, deciden, aportan y participan en la realización del bien común (pág. 2).

La participación ciudadana fortalece los principios democráticos, les da la oportunidad a las personas de expresar sus ideas de forma libre, también 
le aprueba formar parte en la toma de decisiones transcendentales inherentes a su vida cotidiana y que influye en el desarrollo de los procesos sociales.

\section{Metodología}

\subsection{Naturaleza del estudio, tipo y diseño}

Este trabajo investigativo se enmarca bajo los parámetros de la investigación cualitativa. Según Rojas (2011) "es un método de investigación usado principalmente en las ciencias sociales con el propósito de explorar las relaciones sociales y describir la realidad tal como la experimentan los correspondientes" (pág. 1). Se selecciona esta metodología por el tipo de problema, el cual aborda una temática socio ambiental como lo es el saneamiento ambiental y la participación ciudadana; de acuerdo con las características del estudio se seleccionó la modalidad de investigación acción participativa, la cual no solo busca describir la realidad que se observa, sino que busca la transformación de lo observado; en este sentido Pérez (2011) expone:

La Investigación Acción Participativa, es una metodología de investigación que en la actualidad está generando en la Sociedad una mayor capacidad de respuestas a los problemas de dicha Sociedad, ya que el estudio se realiza en la comunidad no solamente por medio de un investigador que estudie la problemática desde afuera, sino que de manera integrada se involucre con cada uno de los miembros de la comunidad, para estudiar los problemas, y las posibles soluciones (pág. 1).

Con la investigación se busca que el investigador se involucre con los sujetos de estudio, es decir que forme parte del colectivo en estudio y participe en el planteamiento de alternativas de solución a los problemas que emergen durante el proceso investigativo. Según Strider (2011): "La investigación descriptiva proporciona un perfil detallado de un evento, condición o situación utilizando métodos ya sea cuantitativos, cualitativos o una combinación de 
ambos" (pág. 1), esta metodología desde el punto de vista narrativo es concebido por Medina (2011): como "un diseño de investigación en el que se recolectan datos sobre las historias de vida y experiencias de ciertas personas para describirlas y analizarlas". En este orden de ideas se fundamenta este método del estudio.

\subsection{Escenario e informantes claves}

La investigación se llevará a cabo en la en la Escuela Nacional Concentrada "Manuela Sáenz", ubicada en la urbanización La Floresta de la parroquia Ciudad Bolivia, Municipio Pedraza del Estado Barinas. Planteado el escenario, se procederá a la selección de los informantes claves los cuales estarán representados por un (1) habitante de la comunidad, un (1) miembro del consejo comunal y un (1) docente que trabaje en la institución. Ellos son los que pueden generar la información necesaria para la realización de la investigación. La información de estas personas es esencial para la elaboración de la propuesta, de allí la importancia de su disponibilidad y franqueza en sus respuestas y sus comentarios.

\subsection{Técnicas e instrumentos de recolección de información.}

La investigación acción participativa es una metodología bastante accesible y puede utilizar una amplia variedad de técnicas investigativas. Para este estudio se utilizará la observación participante combinada con la entrevista en profundidad. Según Grijalva y Mancheno (2012):

La observación puede definirse como el uso que hace el ser humano de los sentidos para recopilar información y comprender el mundo que le rodea. Puede implicar el empleo de todos los sentidos, la valoración y la interpretación de lo que se percibe; todo ello ayuda a dar sentido a la información. En la investigación, la observación constituye un proceso activo en el que se recopilan datos sobre personas, comportamientos, interacciones o acontecimientos, con el objetivo de obtener 
información detallada que pueda ayudar a comprender los fenómenos de estudio (pág. 7).

Con respecto a la entrevista en profundidad Salinas (2010) plantea que" La entrevista en profundidad como técnica de carácter cualitativo y dialógico posibilita acceder en forma privilegiada a los discursos, a los procesos comunicacionales y a la construcción de la cultura" (pág. 1). Para este estudio, la entrevista en profundidad estará orientada por un guion de preguntas semiestructuradas con la finalidad de centrar la conversación en la temática que interesa al investigador. En este sentido también se usará la grabadora para hacer más dinámico el proceso indagatorio y el diario de campo permitirá al investigador realizar cualquier observación acerca de la gestualidad del entrevistado.

\subsection{Presentación y análisis de la información}

La información suministrada es el resultado de la aplicación de las diferentes técnicas de investigación, posteriormente se analizaron los resultados a través de la triangulación, Según Grijalva y Mancheno (2012):

Una de las técnicas de análisis de datos más características de la metodología cualitativa es la "triangulación". El principio básico consiste en recoger y analizar datos desde distintos ángulos para compararlos y contrastarlos entre sí. Denzin (1970: 291) define la triangulación como "la combinación de metodologías en el estudio de un mismo fenómeno." Para Kemmis (1983) consiste en un control cruzado entre diferentes fuentes de datos: personas, instrumentos, documentos o la combinación de todos ellos (pág. 12).

En este sentido, la información que se presentará será el reflejo de la categorización, contrastación y teorización; constituyendo la edificación de un diagnostico profundo y detallado de la situación en estudio, lo que le da a la investigación mayor consistencia y veracidad. 


\subsection{Validez y confiabilidad}

Con relación a la validez Martínez (2006): expresa que "En sentido amplio y general, diremos que una investigación tendrá un alto nivel de "validez" en la medida en que sus resultados "reflejen" una imagen lo más completa posible, clara y representativa de la realidad o situación estudiada". (pág. 6). De acuerdo con lo expuesto anteriormente, esta investigación será validada por el reflejo claro de la realidad que se observe. Con relación a la confiabilidad, el mismo autor afirma que "Una investigación con buena confiabilidad es aquella que es estable, segura, congruente, igual a sí misma en diferentes tiempos y previsible para el futuro" (pág. 8). En tal sentido, el presente estudio demostrara la confiabilidad en la correspondencia de sus resultados, producto de la triangulación de las informaciones recolectadas y de la revisión teórica y el trabajo de campo. La investigación acción participativa permite la utilización de varias técnicas a la vez, lo que fortalece su confiabilidad y la cataloga como uno de las metodologías más estrictas en estudios sociales.

\section{Resultados}

De acuerdo con las respuestas de los entrevistados se evidenció que existe claridad en la conceptualización sobre el saneamiento ambiental, teniendo concordancia con la opinión de los autores antes citados. De esta manera, el trabajo de organización comunal se hace más accesible, ofreciendo alternativas para desarrollar las estrategias en la elaboración de un plan acción para el acondicionamiento higiénico de la Escuela Nacional Concentrada "Manuela Sáenz".

Con respecto a la importancia del saneamiento ambiental, se evidenció que la investigación documental y el aporte de los entrevistados coinciden en que el saneamiento ambiental es importante para mantener un ambiente libre 
de enfermedades; lo cual, demuestra la preocupación común del colectivo por satisfacer las necesidades sanitarias.

Cuando se abordó el tema de los factores de riesgo, los entrevistados identificaron las aguas estancadas como el principal de ellos, opinaron que contribuyen con proliferación de plagas proveedoras de vectores para la propagación de enfermedades contagiosas. En función de esta problemática socioambiental se desarrollarán las acciones en el avance de la investigación.

Como medidas para disminuir los factores de riesgo en la Escuela Nacional Concentrada "Manuela Sáenz" todos los entrevistados acordaron que la mejor manera era la elaboración de un plan acción que contará con la participación de la comunidad y entes gubernamentales. Además debe estar acompañado de un proceso de formación ambiental, fortaleciendo los hábitos higiénicos de la población.

De acuerdo con las diversas fuentes de información se evidenció la gran importancia que tiene la participación comunitaria para resolver problemas comunes que afectan el bienestar de las comunidades. Por esta razón es necesario activar todas organizaciones que promuevan la participación de las personas involucradas con la institución educativa para mejorar sus condiciones ofreciéndoles a los estudiantes un ambiente apto para el aprendizaje integral.

Con las informaciones recolectadas en la revisión documental y las entrevistas realizadas se demostró el interés y la buena disposición de las personas por participar en la elaboración del plan de saneamiento ambiental en Escuela Nacional Concentrada "Manuela Sáenz", manifestando la necesidad de emprender acciones formativas, para concientizar a las personas que habitan alrededor de la institución, también plantean la necesidad de acciones concretas para resolver la problemática abordada. 


\section{Conclusiones}

Con respecto a la fase diagnóstica las personas entrevistadas aportaron información valiosa dentro de las cuales se evidenció la claridad en el conocimiento de las actividades de saneamiento básico, se demostró la preocupación por disminuir los factores de riesgo, concentrando la atención en las aguas estancadas, las cuales son el principal problema tanto para la institución como para la comunidad. Además de identificar los factores de riesgo los entrevistados propusieron la elaboración de un plan acción de saneamiento ambiental, el cual conto con la participación de las personas que hacen vida en Escuela Básica Nacional Concentrada "Manuela Sáenz".

Con relación a la valoración del saneamiento ambiental, se evidenció que la investigación documental y el aporte de los entrevistados coinciden en que el saneamiento ambiental es importante para mantener un ambiente libre de enfermedades; lo cual, demuestra la preocupación común del colectivo por satisfacer las necesidades sanitarias.

Cuando se abordó el tema de los factores de riesgo, los entrevistados identificaron las aguas estancadas como el principal de ellos, opinaron que contribuyen con proliferación de plagas proveedoras de vectores para la propagación de enfermedades contagiosas.

Como medidas para disminuir los factores de riesgo en la Escuela Nacional Concentrada "Manuela Sáenz" todos los entrevistados acordaron que la mejor manera era la elaboración de un plan acción que contará con la participación de la comunidad y entes gubernamentales. Además debe estar acompañado de un proceso de formación ambiental, fortaleciendo los hábitos higiénicos de la población.

En el diseño de la propuesta se consideraron las sugerencias de las personas entrevistadas. El plan de saneamiento ambiental contempló las siguientes fases, en primera instancia se realizaron charlas sobre la importancia de saneamiento ambiental dirigida a los miembros de la 
comunidad. Posteriormente se organizaron las personas en mesas de trabajo para establecer tareas y comisiones para la ejecución del saneamiento ambiental. Luego se planificaron las tareas del saneamiento ambiental.

Para la fase de ejecución del plan acción para el saneamiento ambiental con la participación ciudadana en la Escuela Básica Nacional Concentrada "Manuela Sáenz" se contó con la participación activa de los representantes de la institución, así como también miembros de las organizaciones comunitarias de la colectividad. Los asistentes a la charla mostraron buena atención y concentración en la temática, lo cual permitió al investigador sensibilizar a las personas sobre la importancia del trabajo en equipo para darles un buen ambiente de estudios a los niños y niñas de la institución. Con respecto al resultado de las mesas de trabajo, se evidenció la facilidad que poseen las personas para organizar actividades con un beneficio común; así mismo, en la ejecución de las tareas planificadas, realizaron una satisfactoria jornada de saneamiento ambiental, disminuyendo en sentido los factores de riesgo de la institución.

La realización de esta investigación estuvo sujeta a la participación activa de los padres y representantes de la institución, lo cual evidencia que el estudio demostró la rigurosidad y la confiabilidad de los estudios cualitativos; en este trabajo investigativo los participantes, reconocieron su propia realidad, proponiendo también las alternativas a la solución de sus problemas.

\section{Referencias}

Conferencia Latinoamericana de Saneamiento (2007). Latinos. [Documento en línea]. Recuperado de: http://www.unicef.org/lac/Declaracion de Cali con firmas.pdf

Grijalva, C., y Mancheno, D. (2012). Investigación cualitativa. [Documento en línea]. Recuperado de: 
http://www.monografias.com/trabajos95/investigacioncualitativa/investi gacioncualitativa2.shtm|\#ixzz3NgBMGAkZ

Lake, A. (2007). Agua, medio ambiente y saneamiento. [Documento en línea]. Recuperado de:

http://www.unicef.org/spanish/media/media pr wes.html

Medina, T. (2011). El diseño narrativo. [Documento en línea]. Recuperado de: http://es.slideshare.net/tatis9/diseo-narrativo

Moraga, G. (2011). Constructivismo. [Documento en línea]. Recuperado de: http://www.monografias.com/trabajos11/constru/constru.shtml\#ixzz3U PJ7hbzl

Organización Mundial de la Salud (2014). El saneamiento ambiental y el desarrollo de la higiene. [Documento en línea]. Recuperado de: http://www.who.int/water sanitation health/hygiene/envsan/es/

Pérez, G. (2011). El confinamiento de la basura urbana. [Documento en línea]. Recuperado de:

https://dialnet.unirioja.es/descarga/articulo/4988794.pdf

Strider, C. (2011). Método de investigación descriptivo cualitativo. [Documento en línea]. Recuperado de:

http://www.ehowenespanol.com/metodo-investigacion-descriptivocualitativo-info 386243/

Torrealba, F. (2012) Plan de saneamiento de la Quebrada "Las Piedras" del Barrio Colombia Sur ubicado en el Municipio Guanare del Estado Portuguesa. [Documento en línea]. Recuperado de: http://www.monografias.com/trabajos91/plan-saneamientoquebrada/plan-saneamiento-quebrada2.shtml\#ixzz3PHEb5Z00 


\section{José Antonio Peña Barreto \\ e-mail: joseantbarreto2014@gmail.com}

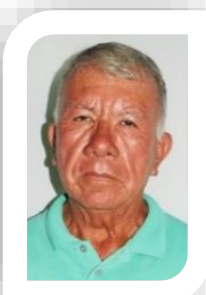

Nacido en Mérida. Estado Mérida. Cursa estudio de Maestría en Educación Ambiente y Desarrollo en la Universidad Pedagógica Experimental Libertador en Barinas Estado Barinas. Licenciado en Educación Integral en la Universidad Nacional Experimental de los Llanos Occidentales "Ezequiel Zamora" (2008). Actualmente se desempeña como docente de aula en La Escuela Nacional Concentrada "Manuela Sáenz" del municipio Pedraza del Estado Barinas durante diez años. 